\title{
La evolución de los marcos (tecno) discursivos del movimiento $15 M$ y sus consecuencias
}

The evolution of (techno) discoursive frames of $15 \mathrm{M}$ movement and its consequences

\author{
EDUARdo Romanos \\ Universidad Complutense de Madrid \\ Igor SÁDABa \\ Universidad Complutense de Madrid \\ igor.sadaba@cps.ucm.es (ESPAÑA)
}

Recibido: 19.062015

Aceptado: 21.09 .2015

\section{RESUMEN}

El movimiento $15 \mathrm{M}$ se caracterizaba, entre otras cosas, por una fuerte crítica a los partidos políticos y el sistema actual de representación política. Sin embargo, el ciclo de movilización ha derivado en un proceso de institucionalización creciente y complejo en el que los activistas del movimiento han participado de manera relativamente visible y continuada en el surgimiento y el desarrollo de nuevos partidos (Partido X, Ganemos y Podemos). Este artículo analiza el papel desempeñado por las nuevas tecnologías en este proceso bajo la hipótesis de que la apropiación de herramientas digitales diseñadas para deliberar, interaccionar o generar formas diversas de participación política en los nuevos partidos reproduce valores y prácticas esenciales del movimiento, allanando con ello el camino para una identificación entre los activistas y los partidos. La mediación tecnológica ha modulado el paso del movimiento al partido al generar un entorno de deliberación horizontal, participación distribuida y estructura descentralizada que reduce las diferencias visibles entre ambos. El desarrollo de las herramientas digitales se ha visto acompañado a su vez de un cambio en los marcos de interpretación de los movimientos sociales con respecto a la tecnología como factor político. De esta forma, las nociones de representación o participación clásicas quedan redibujadas permitiendo una transición suave entre las plazas y los parlamentos. El artículo rastrea la construcción de marcos tecnófilos que, influidos 
por la presencia del software libre y el código abierto, ayudan a entender la organización de los nuevos partidos-movimiento y la transición entre las diversas formas de acción colectiva. Igualmente, se perfilan y describen algunas de estas herramientas y las funciones que han cumplido de cara a forjar unos modelos de organización originales en estos partidos-movimiento.

\title{
PALABRAS CLAVE
}

$15 \mathrm{M}$, nuevos partidos, tecnopolítica, ciclos de protesta, redes sociales.

\begin{abstract}
The Spanish 15M movement (also known as the Indignados) was very critical of political parties and the current system of political representation. However, the protest cycle has evolved into a growing and complex process of institutionalization in which activist have been participating in the creation and development of a number of new parties (Partido X, Ganemos and Podemos). This paper analyzes the role played by new technologies in this process. The basic hypothesis is that the extensive use of digital tools, which encourage participation and deliberation in the creation and development of these new parties, in some ways reproduces essential values and practices of the movement, thus facilitating the identification between the $15 \mathrm{M}$ activists and the parties. In this way, the transition between movement and party has been conditioned by, among other things, a component of technological mediation, generating an environment of horizontal deliberation, participation distributed and decentralized structure. The use of these tools has been accompanied by the development of a certain technophile frame that stresses the potential of new technologies for social and political change. Thus, the classical notions of representation or participation are redrawn allowing a smooth evolution from the squares to the Parliaments. This article examines the construction of technophiles frameworks, which influenced by the presence of free software and open source, help us to understand the organization of the new movement-parties and the transition between the different forms of collective action. Likewise, some of these digital tools are mentioned and outlined and the role that they have played in order to forge new organization models for these movement-parties are described.
\end{abstract}

\section{KEY WORDS}

$15 \mathrm{M}$, new political parties, technopolitics, protest cycles, social media. 


\section{INTRODUCCIÓN ${ }^{1}$}

El movimiento $15 \mathrm{M}$ ha supuesto un auténtico "tsunami sociológico" que ha desafiado algunos de los patrones interpretativos y marcos de comprensión tradicionales de las ciencias sociales. En ese sentido, ha sido ya abordado y estudiado repetidamente a través de sus características más singulares o novedosas: masividad, emocionalidad, humor, descentralización, ausencia de liderazgos visibles, ocupación del espacio público, uso intensivo de las nuevas tecnologías, etc. (para una revisión, véase Nez 2016).

De alguna manera, otro de los rasgos principales del $15 \mathrm{M}$ fue su firme oposición, desconfianza generalizada y fuerte crítica al sistema de partidos y a los modelos de representación política tradicional. Al mismo tiempo, el declive del reciente ciclo de movilización ha deparado un escenario de emergencia de nuevos partidos políticos, afines o cercanos a dicho movimiento. La transición entre el ciclo de movilización y el ciclo electoral ha resultado más rápida y suave de lo que cabría esperar a priori. Este artículo plantea rastrear la mediación tecnológica de ese salto. Es decir, proponemos interpretar el papel desempeñado por el entramado digital y las aplicaciones y plataformas tecnológicas de cara a la evolución de un modelo de movimiento a otro de partido. Como se verá en los discursos de los participantes en los movimientos sociales, la utilización intensiva de cierto tipo de grupos online, redes sociales, apps, webs y sistemas de comunicación virtual ha modulado, e incluso conducido, dicha transformación a través de la creación de estructuras informales, descentralizadas, distribuidas y participativas. El entorno comunicativo generado con ello ha simulado un modo horizontal y abierto de interacción política (un cambio de frame) donde los nuevos partidos se han vislumbrado como continuaciones hasta cierto punto naturales del ciclo de movilización anterior.

Nuestra hipótesis básica es que la tecnología desempeñó un rol más relevante de lo que parece a primera vista en la evolución del movimiento social a los partidos políticos. Lo que esbozaremos en este artículo es que el uso intensivo de herramientas digitales facilita y anima a ciertos modelos de participación descentralizada y deliberación horizontal que fueron fundamentales en la creación de los nuevos partidos. Dichos modos de intervención y acción colectiva tecnológicamente mediada, de alguna manera, reproducen valores y prácticas esenciales del propio movimiento (participación constante, no jerarquías, interacción descentralizada, etc.), facilitando por tanto la identificación entre los activistas del $15 \mathrm{M}$ y los nuevos partidos. No podemos obviar la todavía intensa influencia de los medios analógicos, especialmente periódicos y televisión en la evolución de la acción política en nuestro país

1 Queremos agradecer a Joseba Fernández su colaboración en la realización de las entrevistas y a los dos revisores anónimos sus comentarios y sugerencias, que han contribuido a la mejora del artículo. Por supuesto, cualquier error de apreciación es únicamente responsabilidad nuestra. Este artículo está asociado a los proyectos de investigación ERC Grant 269136 y CSO2013-41035-P financiados por el European Research Council y el Ministerio de Economía y Competitividad, respectivamente

EMPIRIA. Revista de Metodología de Ciencias Sociales. N. ${ }^{\circ}$ 32, septiembre-diciembre, 2015, pp. 15-36. ISSN: 1139-5737, DOI/empiria. 32.2015.15307 
(Rendueles y Sádaba, 2014). Sin embargo, la utilización masiva de aplicaciones informáticas y tecnologías electrónicas, junto con el desarrollo de cierto marco tecnófilo que subraya el potencial de las mismas, ha escoltado la transformación desde el 15M hacia los modos de partido. Es decir, dentro del complejo ecosistema mediático español, los diferentes medios (analógicos y digitales) cubrieron espacios, actuaciones, efectos y acciones diversas en relación a los movimientos sociales.

Sin embargo, intentando también evitar caer en un determinismo tecnológico que asimilaría el uso de ciertos dispositivos tecnológicos al paso directo del movimiento al partido o que soslayaría todo el resto de elementos contextuales (sociales, históricos, culturales, políticos) en la evolución del 15M, no sostenemos aquí que la necesaria deriva institucional del movimiento se explique a partir del uso de Internet y herramientas afines. Más bien, y haciendo uso de un viejo concepto weberiano, estaríamos ante una "afinidad electiva" entre el desarrollo tecnológico (y las esperanzas emancipatorias asociadas al mismo) y ciertos esquemas organizativos del movimiento-partido. La afinidad electiva nos permite explicar los cambios sociales de una manera no determinista sorteando caer en afirmaciones de causalidad fuerte. Weber (2004) aplicó el concepto a la confluencia y reforzamiento mutuo entre la ética calvinista y el capitalismo industrial emergente evitando indicar que uno de ellos era necesariamente la causa preliminar del otro. Aplicándolo a nuestro caso de estudio, podemos decir que los paradigmas interpretativos de la tecnología como recurso social y herramienta política convergen con ciertas formas organizativas. Es decir, los marcos asociados a determinadas prácticas tecnológicas (especialmente aquellas facilitadas o derivadas del mundo del software libre y el open source) serían funcionales a una concepción democratizadora de los dispositivos digitales que acompaña o modula la institucionalización de los movimientos sociales al asimilar ciertas ideas, haciendo equiparable la noción de cooperación descentralizada en red con una organización de partido político horizontal y democrática.

Las fuentes analizadas en este artículo incluyen, principalmente, doce entrevistas en profundidad a activistas tanto del $15 \mathrm{M}$ como de otras organizaciones de movimientos sociales posteriores y/o partidos políticos emergentes (se han utilizado citas para este artículo de solo algunas ellas para una mejor síntesis). Los perfiles comprenden militantes de diferentes edades pertenecientes al 15M, Juventud sin Futuro, Marea Blanca, Marea Verde, Podemos, Ganemos y otras organizaciones políticas. Se ha tratado de cubrir densamente el espacio discursivo de los movimientos sociales y los nuevos partidos a través de militantes tanto de corta experiencia como de largas trayectorias militantes. Los entrevistados han sido seleccionados en calidad de informantes clave por su habilidad para iluminar determinados aspectos de las dinámicas de movimiento social (Blee 2013), en este caso, la institucionalización del $15 \mathrm{M}$ y el papel de las nuevas tecnologías en el proceso. Las entrevistas fueron realizadas en Madrid, Barcelona y Bilbao entre octubre de 2014 y marzo de 2015. La mitad de los entrevistados eran hombres y la mitad mujeres. Las 
entrevistas solicitaban información y la interpretación del entrevistado sobre diferentes procesos de movimiento social, entre ellos la institucionalización. Asimismo, como fuentes de datos se han utilizado documentos, webs, apps, datos de redes sociales, etc.

El artículo se estructura como sigue. Los tres primeros apartados introducen el contexto político, el ciclo de movilización y los nuevos partidos que surgen en el presente ciclo electoral. A continuación se incluye una breve discusión sobre la relación teórica entre movimientos y partidos. Los siguientes apartados exploran la construcción del marco tecnófilo en los movimientos sociales, su aplicación al caso particular de estudio y los usos que vinculan movimientos y partidos al nivel de las prácticas. Todo ello a partir del análisis de las entrevistas mencionadas y de los documentos registrados. Cierran el artículo unas conclusiones que sintetizan los principales hallazgos de la investigación.

\section{CRISIS, DESAFECCIÓN Y DESCONFIANZA}

Los Barómetros del Centro de Investigaciones Sociológicas (CIS) de los años previos a 2011 muestran una creciente desafección de la ciudadanía frente al sistema parlamentario y un deterioro notable de la imagen de los partidos políticos. Ya en octubre y noviembre del 2010, por ejemplo, "la clase política y los partidos políticos" era el principal problema del país para el 18,1\% de los consultados. En diciembre de ese año este dato subía al 19,3\% y 2011 arrancaba con un 20,6\%, niveles en los que se mantuvo (e incluso subió) a lo largo de todo el 2011. La serie histórica sobre la percepción de la situación política española también llevaba acumulando subidas significativas de las categorías "mala" y "muy mala" tal y como se desprende de los resultados que el propio CIS distribuye. En los últimos cuarenta años nunca se habían obtenido valores cercanos al 50\% en la opción "Muy Mala" hasta el año 2013. Como puede comprobarse en los datos del CIS, en apenas 5 años (de 2008 a 2013) la percepción "muy mala" de la situación política nacional subió más de 37 puntos porcentuales (de 11,2\% en noviembre de 2008 a 48,5\% en noviembre de 2013), mientras que la suma de "mala" y "muy mala" lo hizo en más de 44 puntos porcentuales (de 36,8\% en noviembre de 2008 a 81,2\% en noviembre de 2013). Algo que puede visualizarse también en la siguiente gráfica elaborada por dicho organismo: 


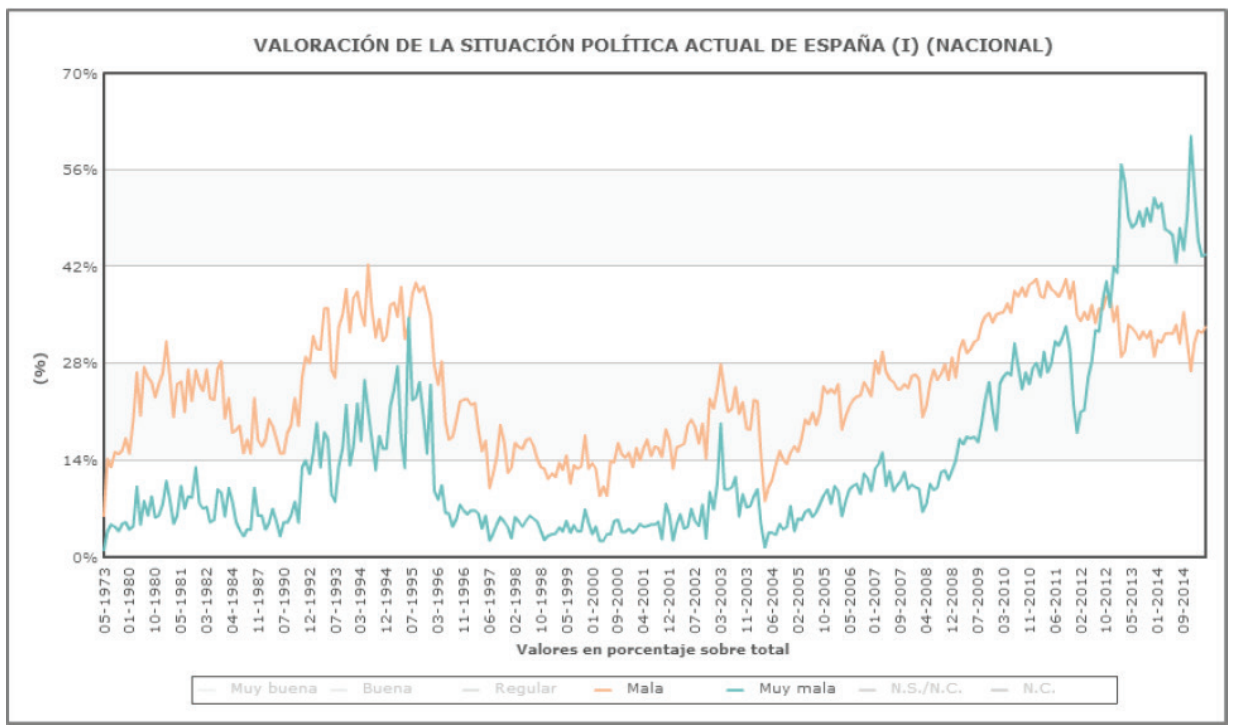

Figura 1: Evolución histórica de las categorías Mala y Muy mala a la pregunta por la situación política española desde 1973. Fuente: Series históricas del CIS (obtenida a través de www.cis.es).

En el contexto en el que se gesta y desarrolla el 15M, gran parte de la población española no solo percibía que la respuesta dada por las autoridades políticas a la gestión de la crisis económica era inadecuada sino que los partidos políticos no funcionaban como mecanismos para manejar la situación de emergencia (Barreiro y Sánchez-Cuenca 2012). Pero, más allá de la imagen erosionada de la clase política, convertida según lo visto en poco tiempo en uno de los mayores problemas percibidos del país, la desconfianza general hacia las instituciones políticas se convirtió en un clima global en niveles locales, nacionales y supranacionales ${ }^{2}$. En general, podríamos atrevernos a asegurar que gran parte del combustible del $15 \mathrm{M}$ provino de una tremenda insatisfacción sobre el funcionamiento general del sistema de partidos. Eslóganes como "no nos representan" o "lo llaman democracia y no lo es" criticaban el modelo de democracia instaurado desde la transición, percibida como una democracia "de baja intensidad" (Arribas 2015) donde las elites políticas luchaban por asegurar el poder de los partidos y sindicatos mayoritarios al mismo tiempo que limitaban el desarrollo de canales de participación de la sociedad civil. Así pues, el ciclo de protesta más masivo en la historia reciente de España se nutrió de ese deterioro manifiesto con respecto al modelo de democracia parlamentaria

2 Cuando hablamos de crisis de la confianza política o desafección no se concluye un proceso general de apatía y desprecio de todas las formas de política (Orriols and Rico 2014: 77-78). Más bien al contrario, entre 2008 y 2011, el interés y la creencia en formas políticas de intervención siguieron la tendencia contraria. Mientras crecía la desafección institucional, la implicación ciudadana en la política en general creció (ibid.; Adell 2011). 
al mismo tiempo que atrajo una amplia simpatía intersectorial por parte de la población en general (Romanos 2013; Sampedro y Lobera 2014). Una gran mayoría de españoles percibía un fuerte déficit democrático al mismo tiempo que demandaba un nuevo sistema político en el que los ciudadanos tuvieran más capacidad de decisión (Font et al. 2012; Font y Alarcón 2012). En este sentido, la corrupción y la percepción del funcionamiento de la política partidista fueron parte importante de la motivación para participar en el 15M, más allá de la crisis económica y su (mala) gestión institucional (Redes, Movimientos y Tecnopolítica 2014; cfr. Likki 2012).

\section{CICLO DE MOVILIZACIÓN E IMPACTOS POLÍTICOS}

Desde mayo de 2011, los indignados desarrollaron un amplio conjunto de acciones: asambleas masivas, acampadas, marchas, manifestaciones, concentraciones, ocupaciones de edificios, escraches, etc. Estas acciones no han provocado, sin embargo, "cambios políticos sustanciales" (Giugni y Bosi 2011) en los procesos de toma de decisiones, procesos legislativos o la apertura de nuevos canales de acceso al sistema político. Así, a pesar de la intensidad y continuidad de las movilizaciones, y del apoyo masivo de los ciudadanos (Sampedro y Lobera 2014), las demandas expresadas por el movimiento no se han visto reflejadas en nuevos textos legales, actuaciones parlamentarias o alteraciones del modelo político en vigor. Dos procesos legislativos muestran con evidencia las limitaciones existentes a este respecto.

El primero es la ley de transparencia, cuya aprobación el gobierno socialista prometió aceleradamente tras la ola de protestas del 15M. Otros grupos políticos aprovecharon igualmente la ocasión para proponer la creación de una comisión parlamentaria para estudiar las demandas del movimiento y "profundizar la democracia y la participación política, además de la transparencia y la rendición de cuentas de las instituciones democráticas"3. En ese momento, España era el único país grande de Europa que no tenía una ley de transparencia y acceso a la información pública. El Consejo de Ministros de 29 de julio de 2011 aprobó un anteproyecto que nunca llegó al Congreso porque ese mismo día se anunció el adelanto de las elecciones (un mes antes el PP había registrado su propia proposición de ley). A cambio, se aceptó una serie de recomendaciones generales no vinculantes entre las cuales se incluían publicar las declaraciones de bienes de los políticos, aumentar el sistema de incompatibilidades y reducir las pensiones de los ex-miembros de las cámaras (Sánchez 2013). Los socialistas perdieron finalmente las elecciones. El 9 de diciembre del 2013, la nueva mayoría conservadora en el parlamento adoptó una "Ley de Transparencia, Acceso a la Información Pública y Buen Gobierno"4 que, sin embargo, colocaba a España por debajo de los estándares internacionales que tanto el 15M como

\footnotetext{
3 Nota de prensa del Congreso de los Diputados, 21/6/2011 (disponible en www.congreso.es).

${ }^{4}$ http://transparencia.gob.es/es_ES/portal-de-transparencia-de-la-age/ley-de-transparencia
} 
buena parte de la sociedad civil habían demandado (Anderica 2013).

El segundo proceso es el relacionado con la Iniciativa Legislativa Popular (ILP) promovida por el movimiento contra los desahucios para paliar el problema de la vivienda en España. La ILP incluía, entre otras medidas, la regulación del sistema de dación en pago, la paralización de los desahucios y la promoción de más viviendas sociales. Aunque se reunieron más de medio millón de firmas, la mayoría conservadora en el parlamento bloqueó la ILP y adoptó, en su lugar, una ley que no incluía gran parte de las demandas anteriores (Romanos 2014).

Más aún, los pocos cambios legislativos que se han producido de facto como respuesta a las movilizaciones han tenido consecuencias negativas para los activistas. Por ejemplo, la recientemente aprobada Ley de Seguridad Ciudadana (también conocida popularmente como "Ley Mordaza") incrementa la cantidad de sanciones y multas para cualquier tipo de protesta y actuación en espacios públicos ya que amplía el rango de acciones punibles e incrementa la severidad de los castigos (Amnesty International 2014). Es decir, más allá del volumen de las movilizaciones, el efecto visible del ciclo $15 \mathrm{M}$ en el sistema político no fue proporcional a la intensidad y el tamaño de las protestas (ni siquiera incluso a la simpatía socialmente recibida). En cierto sentido, se podría afirmar que asistimos a un ciclo radicalmente enfrentado al sistema de partidos y al modelo de democracia representativa tradicional cuyos efectos directos sobre el mismo fueron débiles e incluso insignificantes, al menos desde un punto de vista directo $\mathrm{y}$ evidente en un primer momento.

\section{EL SURGIMIENTO DE NUEVOS PARTIDOS POLÍTICOS}

En los dos epígrafes anteriores hemos perfilado un ciclo de movilización caracterizado por un contexto de alta desafección institucional y descrédito general del sistema de partidos y representación política tradicional y un escaso impacto en el ámbito político. El número, el volumen y la visibilidad de las protestas del ciclo de movilización decrecieron progresivamente, llegando en 2014-2015 a niveles muy por debajo de los alcanzados en años anteriores. El declive del ciclo de protestas ha coincido, sin embargo, con el surgimiento de nuevos partidos políticos de alguna manera vinculados con las movilizaciones en la calle.

El partido más conocido es Podemos, creado en febrero de 2014 por iniciativa de un reducido grupo de profesores de universitarios en colaboración con un pequeño partido político ya existente, Izquierda Anticapitalista, que contribuyó a edificar los cimientos del nuevo partido para terminar subsumido en éste por decisión de su cúpula en un contexto de fuertes tensiones internas (véase Torreblanca 2015; Rivero 2015). Tres meses después de su creación, Podemos consiguió un éxito electoral sin precedentes alcanzando 1,2 millones de votos y 5 eurodiputados en las elecciones al Parlamento Europeo. El éxito de Podemos en las elecciones europeas y el descenso pronunciado de los dos 
partidos mayoritarios (por primera vez por debajo del $50 \%$ de los votos) parece haber abierto una importante brecha en las instituciones políticas. Los vínculos organizativos y sobre todo discursivos de Podemos con los movimientos sociales, y con el 15M en concreto, son palpables y diversos (Martín 2015). Buena parte del grupo promotor original del partido y de la gente que luego ha ido ocupando cargos del responsabilidad dentro del mismo tienen una larga trayectoria de participación en los movimientos sociales y en las organizaciones de la sociedad civil (Flesher 2014; Manetto 2014). A nivel organizativo, el modelo descentralizado y bottom-up de Podemos recuerda la distribución territorial de las asambleas locales del 15M (Martínez y Domingo 2014). La unidad local básica de Podemos han sido los llamados "círculos": agrupaciones de carácter territorial o sectorial que funcionan a través de asambleas. Entre otras funciones, los círculos avalan a los candidatos a cargos de responsabilidad en el partido.

Otro partido proveniente del entorno del $15 \mathrm{M}$ e, incluso más prematuro aunque con menos impacto electoral (100,000 votos en las elecciones europeas), es el Partido $X$, cuyos líderes enfatizan el potencial de la tecnología para fortalecer la democracia participativa y la lucha contra la corrupción (Martínez y Domingo 2014). El partido se presentó públicamente a comienzo de 2013 $\mathrm{y}$, como sus propios miembros reconocen, "la mayor parte de las personas de la Red Ciudadana Partido X, aunque no todas, participamos activamente/nos sentimos del 15M". El partido se define como un "instrumento" al servicio del $15 \mathrm{M}$ en el "terreno electoral" 5 . De hecho, sería "la formación política, tanto en su funcionamiento como en sus aspiraciones, más afín al movimiento" (Lobera 2015: 102; véase también Subirats 2015, Calvo y Álvarez 2015).

Una tercera iniciativa electoral también vinculada con el 15M es Ganemos. $\mathrm{Su}$ creación es más reciente aunque viene precedido de otros proyectos, como Movimiento por la Democracia o Municipalia, que se han ido formando y transformando desde hace tiempo hasta que ha confluido territorialmente formando diversos movimientos municipalistas de diferente cuño. En cada una de las localidades se han ido forjando plataformas de índole variada (Ahora Madrid, Barcelona en Comú, etc.). Mientras que Podemos y el Partido X surgieron como partidos políticos con ocasión de las elecciones al Parlamento Europeo en Mayo de 2014, Ganemos lo ha hecho como una coalición electoral con ocasión de la convocatoria de elecciones municipales de mayo de 2015. $\mathrm{Su}$ ámbito es por tanto más local. De hecho, se presentan como "plataformas municipales" y en ellas caben diversos partidos como los arriba mencionados y otros más antiguos y anteriores al 15M, como Izquierda Unida y Equo. La primera plataforma en crearse fue Guanyem Barcelona en julio de 2014, al frente de la cual se situó Ada Colau, carismática y mediática portavoz de la PAH hasta ese momento con un largo historial de activismo en otras organizaciones y movimientos sociales. Tras Barcelona, diversas plataformas han proliferado por todo el país. Lo que comparten todos estos casos es una dimensión organizativa

\footnotetext{
${ }^{5}$ http://partidox.org.
} 
reticular, agregadora desde lo local, acumuladora de microparticipaciones, sin centros gestores muy visibles, etc.

\section{MOVIMIENTOS, ELECCIONES Y PARTIDOS}

Las elecciones afectan a los movimientos sociales de diversas formas, entre ellas, alterando su estructura de oportunidades (Heaney 2013). Conforme se acerca la convocatoria de elecciones suele crecer el interés de los activistas por utilizar esa plataforma para plantear sus reivindicaciones (Blee y Currier 2006). Los activistas del 15M no han escapado a esa lógica. Muchos de ellos entendieron la convocatoria de elecciones municipales y autonómicas en mayo de 2015 como una apertura de las oportunidades ante la falta de resultados políticos del anterior ciclo de protesta. A ello se suma el impacto de elecciones recientes, en este caso las europeas, y el sorprendente resultado alcanzado por Podemos en ellas, que parece haber afectado la percepción de los activistas con respecto al potencial de la vía institucional:

[Estamos en una] nueva fase que cambia, primero, por la ventana de oportunidad y luego porque la gente se lo está creyendo; se cree que si se llega a ciertas instituciones se va a poder hacer algo. Antes de que surgieran los Ganemos y los Podemos ha habido miles de reuniones y mucha gente de los movimientos sociales pensando cómo acompañamos la movilización de las herramientas institucionales. El estallido llega ahora, pero intencionalidad siempre ha habido. Claramente no son las candidaturas del $15 \mathrm{M}$, tampoco son las candidaturas del ciclo de movilización, pero son candidaturas que solo se pueden entender tras cuatro años de movilización y un bloqueo institucional. Si hubiéramos tenido estos niveles de movilización y se hubieran alcanzado tres o cuatro cosas de las que atacan directamente el corazón del sistema las propuestas institucionales no hubieran tenido tanto éxito. A la gente de los movimientos no nos quedan muchas más [salidas]. A mí no se me ocurre mucho más de lo que hemos hecho y de lo que se está haciendo; qué más podemos hacer para [salir de] una situación de emergencia social como la que se está viviendo en este país. (Entrevista, Hombre, Juventud Sin Futuro, 15M y Podemos)

De algún modo, y este es el quid de la cuestión, la incipiente institucionalización del movimiento $15 \mathrm{M}$ en los partidos arriba reseñados no deja de ser sorprendente dada la fuerte crítica que los activistas plantearon desde el inicio del ciclo de protesta a los partidos políticos y el actual sistema de representación política ("No nos representan"). En este sentido, uno de los resultados de las protestas del 2011 parece ser la aparición de nuevas maneras de articular la relación entre movimientos sociales y partidos políticos. Presenciamos una suerte de movimientos-partido dentro de la cual se observa la evolución entre diferentes formas de acción colectiva.

Las opciones tácticas de los movimientos a menudo incluyen una alta variedad de relaciones con las instituciones (McAdam y Tarrow, 2010). Si 
bien movimientos sociales y partidos políticos son fenómenos distintos que se mueven en niveles diferentes (della Porta y Diani 2011), abundan en la historia casos de confluencia entre estas dos formas de acción colectiva. Los partidos verdes y los partidos socialistas son dos ejemplos de partidos-movimiento que ayudan a entender la evolución histórica del movimiento ecologista y del movimiento obrero, respectivamente. ${ }^{6}$ De manera similar, los nuevos partidos políticos surgidos recientemente en España nos pueden ayudar a entender la evolución del sector de los movimientos sociales en este país. En buena medida, parte de los activistas del 15M entendieron a los nuevos partidos como una nueva táctica del movimiento. Lejos de ser una excepción, McAdam y Tarrow (2010: 165) nos recuerdan que "las tácticas y las estrategias que emplean los grupos de los movimientos sociales incluyen con frecuencia la 'opción electoral". El testimonio del activista incluido anteriormente sostiene que los nuevos partidos españoles son consecuencia de un contexto de enfrentamiento político que va más allá del 15M. En cualquier caso, los activistas del movimiento han entendido que pueden utilizar esos nuevos partidos en su lucha por una democracia más participativa y deliberativa.

Estas agrupaciones han hecho uso simultáneamente de medios de comunicación viejos y nuevos; figuras de líderes carismáticos y organizaciones locales descentralizadas. La combinación entre estas organizaciones y las asambleas y redes comunicativas han generado un paradigma participativo hasta cierto punto novedoso: círculos (Podemos), asambleas (Ganemos) y redes ciudadanas (Partido X). Es decir, estos tres actores políticos han apelado en algún momento a ideas de participación democrática distribuida, redes deliberativas, asambleas públicas, foros políticos, mecanismos horizontales y dispositivos de debate entre iguales. El camino hacia dichos formatos organizativos se ha conformado mediante una serie de discursos (representaciones y formas de interpretar lo digital) y prácticas (herramientas y usos concretos) que describimos a continuación en los siguientes epígrafes.

\section{LA CONSTRUCCIÓN DEL MARCO TECNÓFILO EN LOS MOVIMIENTOS SOCIALES}

La relación de los movimientos sociales con la tecnología ha cambiado históricamente a partir de ciclos de innovación y coyunturas particulares (Tilly y Wood 2012). En general esa relación ha oscilado entre un rechazo frontal inicial (asumiendo que la tecnología no era sino una extensión de las instituciones del poder, el estado o el mercado) hasta una posterior apropiación estratégica e instrumental (optando por la construcción de herramientas alternativas propias). En general, este tipo de respuesta dual ha sido habitual a lo largo de la historia. A cada novedad o invento técnico, los diferentes grupos sociales y

6 Sobre el concepto de partido-movimiento y su aplicación al contexto español actual, véanse Kitschelt (2006) y Martín (2015), respectivamente 
políticos han ido elaborando respuestas y acciones que partían de cierto recelo o rechazo para luego ir incorporándolos gradualmente. Basta ver la llegada del periódico, la radio o Internet y las dimensiones políticas de estas infraestructuras comunicativas (Milan 2013). Pero, en general, durante el largo siglo XX, la tecnología fue percibida mayoritariamente como un ente extraño y externo a los propios movimientos, el resultado del modelo socioeconómico dominante e incluso un instrumento de opresión y explotación (Treré y Barranquero 2013: 29-31). Es lo que podríamos denominar un primer momento tecnófobo, fruto de las lecturas clásicas del capitalismo como productivismo técnico y fabril y de los medios de comunicación como dispositivos de adoctrinamiento o manipulación.

Sin embargo, en los últimos años, la actitud de gran parte de los movimientos sociales hacia las tecnologías digitales ha supuesto un cambio importante. Con la penetración masiva y global de Internet y la entrada en escena del zapatismo y del movimiento antiglobalización se ha generado una predisposición favorable de los movimientos políticos hacia el mundo digital y hacia el terreno comunicativo. Milan (2013) sitúa el cambio a finales de la década de 1990 y el surgimiento de nuevas prácticas políticas precursoras del activismo mediático. La "vida cívica online" (Bennett 2008) o la "Internet politics" (Chadwick 2006) ha alcanzado mayoritariamente a la acción colectiva contemporánea que percibe actualmente las redes sociales y los espacios online como herramientas de intervención política básicas y favorables. Las consecuencias de este salto de la acción offline a la online se han descrito en otros lugares como "virtual brokerage" (Tarrrow y McAdam 2005), "hiperlinked diffusion", "virtual emulation", etc. (Bennett 2004: 206).

Esta situación hace que, en muchos casos, se hable ya de la parte virtual y la parte real de los movimientos sociales, o de sus redes virtuales y redes reales (Diani 2000). En ese sentido se ha desarrollado mucha investigación sobre el impacto de la computer mediated communication (CMC) en la formación de cohesión, solidaridad, identidad colectiva y mayor efectividad de la acción colectiva. Se ha estudiado también la formación de redes trasnacionales, la gestión de nuevos recursos en este tipo de comunidades, nuevos repertorios de acción, originales formatos de movilización, etc. (Kelly Garret 2006). Sin embargo, no siempre se ha descrito adecuadamente la manera en que ciertos cambios en los marcos interpretativos de los movimientos sociales (Benford y Snow 2000) han permitido la apropiación efectiva de dichos recursos tecnológicos. Es decir, las transformaciones en la manera de interpretar el potencial político de la tecné digital y cómo ello ha alterado la forma de actuación de dichos movimientos. Para que los grupos ciudadanos hayan podido asumir estratégicamente la utilización de las tecnologías se ha tenido que producir previamente un cambio de actitud favorable a ello; un cambio de paradigma interpretativo dispuesto a asumir los beneficios de dicho paso. ${ }^{7}$

${ }^{7}$ Lewis Munford (2010) explicaba de manera similar la evolución de la innovación tecnológica. Evitando cualquier modelo explicativo determinista hacía hincapié en los cambios culturales que predisponen a la adopción, uso y extensión de ciertos aparatos o dispositivos técnicos, repre-

EMPIRIA. Revista de Metodología de Ciencias Sociales. N. ${ }^{\circ}$ 32, septiembre-diciembre, 2015, pp. 15-36. ISSN: 1139-5737, DOI/empiria. 32.2015.15307 
Este "technological turn" implica un cambio de frames en la definición y comprensión de la acción colectiva. Hoy en día, las tecnologías digitales forman una suerte de ecosistema donde los movimientos sociales se sienten cómodos. Se podría decir que las representaciones sociales de los movimientos sociales están decantándose hacia una aceptación de las mismas e, incluso, hacia cierta tecnofilia. Han surgido movimientos centrados en objetos tecnológicos (el software libre) o movimientos muy dependientes e impensables sin la tecnología (antiglobalización, 15M, primavera árabe, Occupy Wall Street, etc.). En tales circunstancias, la tecnología se percibe como una "oportunidad" (Van Laer y Van Aelst 2010), como un recurso necesario y básico dada la mediatización general del mundo social. En una época de medios globales, la tecnología digital comienza a entenderse como una herramienta básica para coordinarse, visibilizarse, conectar grupos y construir identidades debido a su bajo coste e inmediatez, el surgimiento de audiencias mundiales, la no dependencia de la geografía y el anonimato estratégico. Los nuevos marcos tecnófilos han interpretado lo técnico como una herramienta útil, apropiable, configurable y de alto poder transformador (Carpentier 2011).

El desplazamiento de la acción colectiva no convencional a la institucional al que antes hacíamos alusión se ha vehiculado, entre otras formas, mediante una noción de participación constante conducida y articulada a partir de las tecnologías digitales. De hecho, algunos analistas han identificado en el uso creativo de las nuevas tecnologías para estimular la participación uno de los elementos más claros de continuidad entre las dos formas de acción colectiva: movimiento $15 \mathrm{M}$ y nuevos partidos políticos (Lobera 2015; Calvo y Álvarez 2015). En general, los nuevos partidos colaboran en la construcción de un marco de interpretación de un nuevo modelo democrático que evitaría caer en los errores que los partidos políticos tradicionales han cometido. En este sentido, la diferencia entre movimiento y partido se desdibuja en un partido que se organiza al revés que las instituciones tradicionales (ya muy criticadas y deslegitimadas). Las redes sociales contribuirían a dislocar la dinámica tradicional de partido jerárquico hacia un esquema de funcionamiento democrático, "desde-abajo" y donde la ciudadanía realmente elige o marca el debate.

Estaríamos ante un nuevo "ethos tecnológico" (Hakkinen 2004) muy vinculado al mundo del software libre y de la open culture. De alguna manera, las libertades formales de participación en el proceso de construcción tecnocientífico de dichos movimientos vendrían a coronar una idea de apertura y horizontalidad muy influyente. Gran parte de los movimientos sociales de finales del siglo $\mathrm{XX}$ ya no se ven influenciados por paradigmas de frustración o protestas estilo NIMBY sino más bien por esquemas interpretativos cercanos a la idea de cultura abierta y a la participación descentralizada mediante procedimientos tecnológicos. Las referencias históricas de casos como Linux, Wikipedia e incluso Wikileaks han sido guías de actuaciones políticas ejemplares donde el acceso abierto, la rendición de cuentas, el uso de las redes

sentaciones sociales y actitudes sin las cuales ninguna tecnología prosperaría. 
digitales y la cooperación descentralizada han funcionado con cierto grado de éxito (Sampedro 2014). Todos estos fenómenos se han ganado habitualmente la etiqueta de democratizadores: del saber técnico, del conocimiento humano, de la información política, etc. Pero a la vez, sus valores están asociados a su metodología organizativa, arquitectura técnica, procesos de cooperación, transparencia, etc. Aunque no se haya producido un trasvase o difusión muy explícito hacia los casos que estudiamos aquí, las huellas y rastros de estos paradigmas culturales son evidentes. Por ejemplo, el Partido X hace múltiples referencias a estos valores y en su Hoja de ruta menciona 8 veces el "software", 17 veces la "tecnología", 13 veces la noción de procedimiento "abierto" y 26 veces la idea de "libertad" o "libre". Por no mencionar el vocabulario que aparece en su organización: "nodos", "matriz", "red", "I+D+X”, etc. En cambio no aparece la palabra "izquierda" o "movimiento social" en ningún momento 8 . Esta nueva cultura participativa y del entorno 2.0 sirve como fundamento discursivo a nuevos modelos de democracia institucional y organización de partido (Papacharissi 2008). A partir de ahora, el software libre será una experiencia o imagen a imitar.

\section{EL MARCO TECNÓFILO EN LOS PARTIDOS-MOVIMIENTO EN ESPAÑA}

Como se ha avanzado más arriba, los nuevos marcos tecnológicos asumidos por los partidos del ciclo post-15M no solo desarrollan nuevas versiones de participación política sino que integran también un nuevo concepto de representación. Se pasa de la representación política delegada (clásica) a la representación distribuida (las redes descentralizadas y cooperantes como formas de representar la ciudadanía). Los modelos de participación deliberativa y reticular de Internet ejemplificarían una versión o esquema más horizontal y asambleario (consultas continuas) que generan una sensación de votación constante o referéndum permanente (Gimmler 2001). De esta forma, el imaginario tecnológico donde las redes digitales se pueden interpretar como manos invisibles para una agregación coherente y eficiente de preferencias y opiniones ejercería un efecto determinante. Incluso podríamos llegar a decir que las redes tecnológicas descentralizadas "simulan" una democracia real, algo que reconocen los activistas valorando este simulacro como un experimento político relevante con efectos reales:

"Creo que las redes sociales, porque justamente son redes y son horizontales y son accesibles, han sido el espacio donde hemos simulado o hemos querido simular ese espacio de participación ciudadana radicalmente masivo en el que poder experimentar. Creo que justamente ha sido el laboratorio donde hemos generado muchísimas de las propuestas sobre cómo queremos hacer democracia en el futuro. Ha sido fundamental, fundamental." (Entrevista, Mujer, 15M y

\footnotetext{
${ }^{8}$ http://partidox.org/pdf/hoja-de-ruta-europa.pdf
} 


\section{Ganemos)}

Idénticamente, el uso de las tecnologías digitales ha reconceptualizado incluso las nociones clásicas de política y democracia, su temporalidad y construcción. De alguna manera, los espacios virtuales son "opinódromos" constantes donde la mera conversación (una gran conversación colectiva) se percibe como un nuevo tejido sociopolítico:

"Yo creo, por un lado, que el tema de las redes sociales nos ha permitido hacer y entender que la política ocurre todos los días, ¿no? No reducir la política a eventos, sino que_lo cotidiano también es político, y tiene una carga política que tiene que ser entendida, interpretada y denunciada." (Entrevista, Mujer, 15M y Ganemos)

"Para luchar se entiende que los sindicatos no sirven, que hay que hacer algo distinto y eso se mezcla con el uso de las redes sociales, que yo creo que han jugado un papel enorme. Pues aparecen perfiles de Facebook, grupos de whatsapp, etc. donde se discute de política, donde se crea opinión, donde nadie es más que nadie. (...) Hoy en día el desarrollo técnico e informático lo que hace es que favorece mucho esa cultura de estar constantemente opinando, de qué nadie es más que nadie. Me parece que esa voluntad democrática rompe mucho con el tema de la representatividad en la medida que el desarrollo técnico lo posibilita (Entrevista, Hombre, Marea Blanca).

Las tecnologías han cumplido un papel de simulación, emulación o representación de una construcción no jerárquica, democrática y participativa. A tal punto llega esta noción, que los activistas entrevistados asociaban las redes sociales a "nuevas formas democráticas" y visibilizaban Internet como un espacio político:

"Esta idea de que hay dispositivos muy poco democráticos que producen mucha democracia. Hay algo en esa lógica como multicapa y en las reverberaciones que dispositivos poco transparentes o poco participados generan participación que es algo interesante a pensar. ¿Es democrático cómo se gestionaba la cuenta de Twitter de Acampada Sol? En absoluto. ¿Producía democracia? Infinita. Yo creo que tiene que ver, por un lado, como con la producción de una creencia de posibilidad que te activa, ¿no? Y por otro lado, con la capacidad de producir relaciones." (Entrevista, Hombre, movimiento antiglobalización, $15 \mathrm{M}$ y Ganemos)

No en vano, estamos ante movimientos-partidos que han eclosionado en el momento en el que Internet se vislumbra como un medio ubicuo y eficaz para la comunicación cosmopolita (Kelty 2008). En este sentido, los movimientos sociales construyen una idea de las redes digitales como espacios de apropiación, como lugares de actuación y como medios autónomos y libres (Treré y Barranquero 2013). En esta línea, en la opinión de los responsables de Podemos, la tecnología no solo aporta visibilidad al partido, que sería el uso que los partidos políticos tradicionales han venido haciendo de las nuevas tecnologías; 
también genera comunidad. Finalmente, se puede observar cómo los usos que los movimientos sociales hacen de las redes digitales se han ido "trasladando" hacia el modelo electoral de partido político:

"Las redes son una especie de vivero en el que ha crecido Podemos. Hemos roto los esquemas que utilizaban habitualmente los partidos y hemos tratado de generar una comunidad de personas que sintiera que podía participar a través de las redes sociales, comentar, dar su opinión, participar en los procesos internos... de alguna manera aportar su grano de arena. Nos inspiramos siempre en el modelo de funcionamiento en redes sociales que han tenido los movimientos sociales en los últimos años y lo fuimos trasladando a una herramienta que servía para participar en unas elecciones... Vamos a ir creando una especie de engranaje para que la gente que participaba de forma espontánea lo haga ahora de forma ordenada." (Moreno 2014).

\section{HERRAMIENTAS TECNOLÓGICAS Y PRÁCTICAS COMUNICATIVAS EN LOS NUEVOS PARTIDOS}

No solo existe una percepción y expresión discursiva del papel político de lo tecnológico sino también una serie de prácticas tecnopolíticas concretas. En los tres principales partidos surgidos tras el 15M (Podemos, Partido X y Ganemos) encontramos una apropiación diferencial de las herramientas digitales. El uso de diferentes apps, software o dispositivos tecnológicos es muy variado, yendo desde las ya "clásicas herramientas" (redes sociales, Facebook y Twitter fundamentalmente y algo de Youtube) hasta auténticas novedades o especificidades (aplicaciones propias diseñadas particularmente para actividades puntuales y software de muy reciente aparición que mencionaremos a continuación). En función de dónde o a través de qué tipo concreto de aplicaciones se apoya cada organización podemos intentar interpretar la relación de cada una de ellos con las tecnologías digitales. Así, mientras Podemos ha ensayado con nuevas herramientas tecnológicas que existían ya en el espacio comercial (Reddit, Appgree, etc.), pero que apenas eran usadas por movimientos sociales, el Partido $\mathrm{X}$ ha tratado de desarrollar las suyas propias y Ganemos ha preferido quedarse en las tradicionales (Facebook, Twitter y Youtube). En ese sentido, la variabilidad en los discursos, posiciones y usos de los medios sociales compone un mapa de partidos-movimiento más complejo de lo que podría pensarse en un primer momento.

Desde el punto de vista de lo que son las redes sociales online "clásicas" encontramos diferencias significativas en el tamaño de las comunidades que se han compuesto alrededor de estas organizaciones. Aun así, los tres casos tienen presencia en dichas redes (grupos de Facebook, cuentas de Twitter y canales en Youtube): 


\begin{tabular}{|l|c|c|c|}
\hline & Facebook & Twitter & Youtube \\
\hline Podemos.info & 916.195 likes & 472.119 followers & $\begin{array}{c}156 \text { videos, } \\
44.579 \\
\text { suscripciones }\end{array}$ \\
\hline $\begin{array}{l}\text { PartidoX.org } \\
\text { (Red ciudadana) }\end{array}$ & 85.900 likes & 45.749 followers & $\begin{array}{c}95 \text { videos } \\
3.353 \\
\text { suscripciones }\end{array}$ \\
\hline $\begin{array}{l}\text { Ganemosmadrid. } \\
\text { info (solo Madrid) }\end{array}$ & 8.214 likes & 19.819 followers & $\begin{array}{c}13 \text { videos } \\
\text { suscripciones }\end{array}$ \\
\hline
\end{tabular}

Elaboración propia a partir de datos obtenidos el 25/12/2014.

El uso de Facebook y Twitter no deja de ser una fuente inagotable y sugerente de datos, tanto para el $15 \mathrm{M}$ como para otros movimientos recientes (véase Lotan et al. 2011; Bennet y Segerberg 2011). Estas redes sociales han sido consideradas desde disparadores o catalizadores hasta auténticos elementos estructurales y organizativos de las propias protestas (véase, por ej., Harlow 2012; Choi 2014). En nuestro análisis lo más relevante es la diferencia que aparece en el caso de Facebook (likes a la página) a favor de Podemos en relación con Twitter (followers) y con Youtube (suscripciones).El hecho de que la parte de microblogging y videos online no es tan diferente en comparación con la gran red social (Facebook) parece un indicador empírico notable de la diferente cantidad de seguidores y militantes de cada grupo. No obstante, estas métricas son siempre aproximadas de las audiencias y al no estar construidas bajo conceptos teóricos propios de las ciencias sociales no tienen por qué ser directamente proporcionales al tamaño de una organización, la cantidad de movilizaciones que convoca, su visibilidad pública o el éxito de sus demandas. Nos es útil aquí para mostrar la presencia online de los tres casos analizados y evidenciar que siguen siendo espacios de fuerte presencia política.

Sin embargo, lo interesante es que además de las redes sociales u otras apps habituales, estos nuevos partidos han utilizado, adaptado o implementado nuevas herramientas que, hasta la fecha, no eran tan frecuentes en los movimientos sociales o en los partidos políticos. Resumimos en el siguiente cuadro los tres partidos con sus herramientas: 9

9 Una descripción de algunas de estas herramientas, en Perez-Lanzac (2015), Gutierrez-Rubí (2014) 


\begin{tabular}{|c|c|c|c|}
\hline PARTIDO & $\begin{array}{l}\text { HERRAMIENTAS } \\
\text { PRINCIPALES }\end{array}$ & FUNCIONES & ACCESO \\
\hline PODEMOS & $\begin{array}{l}\text { Reddit, Appgree, } \\
\text { Loomio, Agora } \\
\text { voting, Titan Pad }\end{array}$ & $\begin{array}{l}\text { Debate, trabajo } \\
\text { colaborativo, } \\
\text { sondeos y } \\
\text { votaciones, } \\
\text { trabajo en } \\
\text { grupo }\end{array}$ & $\frac{\text { https://podemos.info/ }}{\text { participa/herramientas/ }}$ \\
\hline $\begin{array}{l}\text { PARTIDO } \\
\quad \mathrm{X}\end{array}$ & $\begin{array}{l}\text { BarX, AgendaX, } \\
\text { BuzónX }\end{array}$ & $\begin{array}{l}\text { Encuentro, } \\
\text { d e b a t e, } \\
\text { seguimiento, } \\
\text { colaboración }\end{array}$ & $\begin{array}{l}\text { http://partidox.org/como- } \\
\text { participar/ } \\
\text { http://partidox.org/ } \\
\text { informacion-basica/ }\end{array}$ \\
\hline GANEMOS & $\begin{array}{l}\text { Hoja de ruta y } \\
\text { Cron o g ram a, } \\
\text { Agenda }\end{array}$ & $\begin{array}{l}\text { Intercambio, } \\
\text { planificación }\end{array}$ & $\begin{array}{l}\text { http://ganemosmadrid.info/ } \\
\text { grupo-de-comunicacion/ }\end{array}$ \\
\hline
\end{tabular}

Tabla 2: partidos, herramientas y funciones. Elaboración propia.

En general, podemos clasificar estas herramientas en tres grandes grupos:

i) de interacción, debate y deliberación;

ii) de edición, colaboración y creación;

iii) de información y difusión.

Estos grupos se corresponden con las principales funciones del espacio tecnológico: i) construcción identitaria, ii) producción material y iii) visibilización, respectivamente. Cada grupo, no necesariamente homogéneo, prioriza algunas funciones sobre otras. Pero las tres ayudan a dar cohesión y sensación de unidad al movimiento-partido, lo estructuran de alguna manera, no tanto como aparato institucional sino a partir de un sentimiento de pertenencia conjunta y de cooperación masiva. Ese conjunto de representaciones de unidad, catalizadas mediante las herramientas tecnológicas, facilita la evolución y consolidación de este tipo de organizaciones.

La idea general que subyace tras el uso de todas las aplicaciones arriba descritas es que la mayoría de ellas, de alguna manera, garantizan un mayor nivel de participación directa de la población en general, gracias a las potencialidades y características del entorno tecnológico. $\mathrm{O}$, al menos, tal y como se vio en las entrevistas, generan esa sensación de verse involucrado mediante mecanismos horizontales y democráticos en un proyecto. En tal caso, esa mayor "participación" generalizada (real o percibida) sería una condición para desarrollar un modelo de democracia directa (digital pero con efectos reales), eliminando los estereotipos negativos que los partidos políticos clásicos (verticales y más autoritarios) podrían tener. Dicho de otra forma, el paradigma de participación online rompe las barreras de las reticencias e imágenes 
negativas sobre las jerarquías de los partidos políticos, favoreciendo o facilitando la transformación de los movimientos sociales hacia modelos organizativos electorales pero manteniendo intactos sus principios de democracia de base y horizontalidad.

\section{CONCLUSIONES}

Este artículo ha tratado de abordar un fenómeno que, hasta la fecha, no ha sido tratado en profundidad: la relación y transición entre el $15 \mathrm{M}$ y los nuevos partidos emergentes surgidos posteriormente de su entorno. Más en concreto, lo que se ha intentado mostrar es que, frente a la idea inicial de rechazo y enfrentamiento entre la indignación popular y el sistema de representación política, más bien se ha generado una transición suave y nada traumática de un modo de acción colectiva a la otra. A pesar de un grado inusualmente alto de desafección y desconfianza institucional, el resultado del ciclo post-15M ha sido la aparición de más partidos políticos. Dicha evolución desde las movilizaciones del 2011 hasta los partidos emergentes, aunque no fue prevista inicialmente, se ha producido de una manera natural y superando las connotaciones negativas de los partidos clásicos (jerarquía, corrupción, autoritarismo, verticalidad, etc.). Para ello, se ha hecho uso, tal y como se ha visto en este trabajo, tanto de ciertos marcos discursivos como organizativos cercanos al mundo de las tecnologías digitales. El modelo de software libre, abierto, descentralizado, reticular y distribuido ha funcionado como esquema de referencia para un tipo de organización que ha retenido elementos de un movimiento social a pesar de convertirse en partido político (a través de círculos, asambleas, redes o colectivos locales en constante debate). Para ello, han mediado un conjunto de representaciones sociales, imágenes y discursos, algunos de los cuales hemos intentado mostrar, apoyándonos en una serie de entrevistas abiertas a militantes y activistas, entre otras fuentes. Dichos modelos organizativos y perceptivos han estimulado la aceptación del modo partido al identificar en los espacios digitales una continuidad entre las virtudes de los movimientos sociales (descentralización, democracia, participación constante y política cotidiana, ausencia de jerarquías, opinión continua, debate enriquecedor, etc.) y los nuevos partidos. Igualmente, se ha tratado de visualizar que el uso de redes sociales y de nuevas plataformas y apps con propiedades muy particulares y concretas ha jugado un papel también relevante en este aspecto.

\section{BIBLIOGRAFÍA}

ADELL, R. (2011): "La movilización de los indignados del 15-M. Aportaciones desde la sociología de la protesta”, Sociedad y Utopía 38: 141-170.

ANDERICA, V. (2013): "Una ley de transparencia que no nos saca de la opacidad", El Diario.es. http://www.eldiario.es/zonacritica/ley de transparencia-opacidadsilencio administrativo-derechos fundamentales 6 174342577.html 
AMNESTY INTERNATIONAL (2014) "Spain: The Right to Protest Under Threat". http://www.amnesty.org/en/library/info/EUR41/001/2014/en.

ARRIBAS, A. (2015) "Recordar el 15M para reimaginar el presente. Los movimientos sociales en España más allá del ciclo electoral de 2015”, Interface 7(1): 150-164.

BARREIRO, B. SÁNCHEZ-CUENCA, I. (2012): "In the Whirlwind of the Economic Crisis: Local and Regional Elections in Spain", South European Society and Politics 17(2): 281-294.

BENFORD, R. Y SNOW, D. (2000): "Framing Processes and Social Movements: An Overview and Assessment", Annual Review of Sociology 26: 611-639.

BENNETT, L. (2004): "Social movements beyond borders: understanding two eras of transnational activism" en Transnational Protest and Global Activism, Oxford, Rowman \& Littlefield.

BENNETT, L. (2008): Civic Life Online. Learning How Digital Media Can Engage Youth, The MIT Press.

CALVO, K. E I. ÁLVAREZ (2015): Limitaciones y exclusiones en la institucionalización de la indignación: del 15-M a Podemos, Revista Española de Sociología 24: 115-122.

CARPENTIER, N. (2011): Media and participation. A site of ideological democratic struggle. Chicago, The University of Chicago Press.

CHADWICK, A. (2006): Internet politics: States, citizens, and new communication technologies, Oxford, Oxford University Press.

CHOI, S. (2014): “An exploratory approach to a Twitter-based community centered on a political goal in South Korea: Who organized it, what they shared, and how they acted", New Media \& Society 16: 129-148.

DELLA PORTA, D. Y DIANI, M. (2006): Social Movements: An Introduction ( $2^{\text {nd }}$ ed.), Malden, Blackwell.

DIANI, M. (2000): "Social movement networks virtual and real", Information, Communication \& Society 3(3): 386-401.

FLESHER FOMINAYA, C. (2014): "Spain is Different": Podemos and 15-M, Open democracy, 29 May.

FONT, J. Y ALARCÓN, P. (2012) “CCómo queremos que se tomen las decisiones políticas", Zoom Político 2.

FONT, J., NAVARRO, C., WOJCIESZAK, M. Y ALARCÓN, P. (2012): “"Democracia sigilosa» en España? Preferencias de la ciudadanía española sobre las formas de decisión política y sus factores explicativos, Madrid: CIS.

GIMMLER, A. (2001): "Deliberative Democracy, the Public Sphere and the Internet", Philosophy and Social Criticism 27(4): 21-39.

GIUGNI, M. Y BOSI, L. (2011): "The Impact of Protest Movements on the Establishment: Dimensions, Models, and Approaches" en The 'Establishment' Responds: Power, politics and protest since 1945, New York, Palgrave Macmillan, pp. 17-28.

GUTIERREZ-RUBÍ, A. (2014): “Micropolítica”, El País, 26 octubre 2014. http://blogs. elpais.com/micropolitica/2014/10/tecnopolitica.html

HARLOW, S. (2012): "Social media and social movements: Facebook and an online Guatemalan justice movement that moved offline", New Media \& Society 14(2): 225-243.

KELLY GARRETT, R. (2006): "Protest in an information society: A review of literature on social movements and new ICTs", Information, Communication \& Society 9:2, 202-224. 
KELTY, C. (2008): Two Bits: The Cultural Significance of Free Software and the Internet Durham, Duke University Press.

KITSCHELT, H. (2006): Movement Parties, en Handbook of Party Politics, Londres, Sage, pp. 278-290.

LIKKI, T. (2012): “15M Revisited: A Diverse Movement United for Change". ZoomPolitico 11.

LOBERA, J. (2015): De movimientos a partidos. La cristalización electoral de la protesta, Revista Española de Sociología 24: 97-105.

LOTAN, G. ET AL. (2011): "The Revolutions Were Tweeted: Information Flows during the 2011 Tunisian and Egyptian Revolutions", International Journal of Communication 5: 1375-1405.

MANETTO, F. (2014): "El círculo de confianza de Iglesias", El País, 16 Noviembre.

MARTÍN, I. (2015): Podemos y otros modelos de partido-movimiento, Revista Española de Sociología 24: 107-114.

McADAM, D. Y TARROW, S. (2010): "Ballots and Barricades: On the Reciprocal Relationship between Elections and Social Movements", Perspectives on Politics 8(2): 529-542.

METROSCOPIA (2013): Clima social de España (43a oleada), 11 Enero.

MILAN, S. (2013): Social Movements and Their Technologies. Wiring Social Change. Londres, Palmgrave MacMillan.

MILAN, S. Y HITZ, A. (2013): "Networked Collective Action and the Institutionalized Policy Debate: Bringing Cyberactivism to the Policy Arena?" Policy and Internet 5(1): 7-26.

MORENO, M. (2014): "Podemos: `El resto de los partidos no se ha tomado en serio las redes sociales",, Cuarto Poder, 10 Diciembre. http://www.cuartopoder.es/ dospuntocero/2014/12/10/podemos-el-resto-de-lo-partidos-no-se-ha-tomado-enserio-las-redes-sociales/1584

MUNFORD, L. (2010 [1934]): Technics and Civilization, Chicago, University of Chicago Press.

NEZ, H. (2016): "Social Movements in Times of Crisis: Indignados and Occupiers in Europe" en Social Movement Studies in Europe: The State of the Art, Nueva York, Berghahn.

ORRIOLS, L. Y RICO, G. (2014): “El clima de opinión” en Elecciones generales 2011, Madrid, CIS, pp. 63-82.

PAPACHARISSI, Z. (2008): “The Virtual Sphere 2.0: The Internet, the Public Sphere and beyond" en The Routledge Handbook of Internet Politics, New York, Routledge.

RENDUELES, C. Y SÁDABA, I. (2014): La hipótesis ciberpolítica. Una aproximación crítica, Documentacion Social 173, 95-116.

REQUENA, A. Y PICAZO, B. (2013): La pobreza laboral aumenta en España, eldiario.es, 2 Abril, http://www.eldiario.es/economia/pobreza-laboral-aumentaEspana_0_115488999.html

RIVERO, J. (2015): Podemos. Objetivo: asaltar los cielos, Barcelona, Planeta.

ROMANOS, E. (2013): "Collective Learning Processes Within Social Movements: Some Insights into the Spanish 15-M/Indignados Movement" en Understanding European Movements: New Social Movements, Global Justice Struggles, AntiAusterity Protest, London: Routledge, 203-219.

ROMANOS, E. (2014): Evictions, Petitions and Escraches: Contentious Housing in Austerity Spain, Social Movement Studies 13(2): 296-302. 
SAMPEDRO, V. Y LOBERA, J. (2014): "The Spanish 15-M Movement: a consensual dissent?", Journal of Spanish Cultural Studies 14(4): 1-20.

SÁNCHEZ, J. L. (2013): Las 10 mareas del cambio, Barcelona, Roca.

SUBIRATS, J. (2015): Todo se mueve. Acción colectiva, acción conectiva. Movimientos, partidos e instituciones, Revista Española de Sociología 24: 123-131.

TARROW, S. Y McADAM, D. (2005): "Scale shift in Transnational Contention" Trasnational Protest and Global Activism, Rowan \& Littlefield, pp. 121-150.

TILLY, C. Y L. WOOD (2012): Social Movements, 1768-2012, Boulder, Paradigm.

TORREBLANCA, J.I. (2015): Asaltar los cielos: podemos o la política después de la crisis, Barcelona, Debate.

TRERÉ, E. Y BARRANQUERO, A. (2013): “De mitos y sublimes digitales: movimientos sociales y tecnologías de la comunicación desde una perspectiva histórica", Revista de Estudios para el Desarrollo Social de la Comunicación 8: 27 47.

VAN LAER, J. Y P. VAN AELST (2010): "Internet and social movement action repertoires. Opportunities and limitations", Information, Communication \& Society 13(8): $1146-1171$.

WEBER, M. (2004 [1905]): La ética protestante y el espíritu del capitalismo, Madrid, Alianza. 